\title{
An Improved Adaptive Smoothing Method
}

\author{
Xin $\mathrm{Hu}^{1}$, Hui Peng ${ }^{2}$, Joseph Kesker ${ }^{3}$, Xiang Cai ${ }^{4}$, William G. Wee ${ }^{4}$, \\ and Jing-Huei Lee ${ }^{5}$ \\ ${ }^{1}$ Microsoft Corporation \\ Redmond, Washington, United States, 98052 \\ \{huxin82\} @gmail.com \\ 2 Advantest America, INC \\ Santa Clara, California, United States, 95054 \\ $\{\mathrm{h}$.peng $\}$ advantest.com \\ ${ }^{3}$ Sheet Dynamic Ltd., \\ Cincinnati, Ohio, United States, 45212 \\ \{jmkesler\}@gmail.com \\ ${ }^{4}$ Department of Electrical and Computer Engineering, \\ University of Cincinnati, Cincinnati, Ohio, United States, 45221 \\ \{caixg, wwee $\}$ @ececs.uc.edu \\ ${ }^{5}$ Department of Biomedical Engineering, \\ University of Cincinnati, Cincinnati, Ohio, United States, 45221 \\ \{leej8\} @ucmail.uc. edu
}

\begin{abstract}
An improvement of the Chen's method has been provided through the calculation of a more accurate $\mathrm{H}$ map. The $\mathrm{H}$ map is the pixel's contextual inhomogeneity value reflecting its proximity position with respect to an edge feature, and a more accurate $\mathrm{H}$ value leads to the more accurate smoothing speed for the pixel. While experiments on 5 real images show slight improvements in SNRs of our method over that of the Chen method, edge features preserving capability has been enhanced with low FARs (false alarm rates) for edge feature extracted from applying the Sobel filter to the image. Furthermore, parameter values have been determined through an exhaustive searching process resulting in the suggestions of $\mathrm{h}=0.4$ and $\mathrm{T}=4$ for practical applications where the original noise free image is not available and/or no viewer to visually make a selection of the final smoothed image as the output.
\end{abstract}

\section{Introduction}

Image smoothing is an image enhancement method with the objective of reducing and/or removing image noise. In a general case, it is impossible to completely remove image noise and recover the original noise free image. Generally, the image smoothing algorithm will blur the image edge features while removing the image noise [3].

In smoothing operations, images are assumed to have an approximately piecewise constant gray level distribution [2,3]. Based on this widely adopted assumption, an image can be viewed as the combination of many local regions with constant intensity. The separations of these local regions are edge features comprised of connected edge pixels. These edge features are of great importance in characterizing 
image content. Therefore, a desirable quality of an image smoothing algorithm is to preserve image edge features while removing the image noise. Most of the current image smoothing algorithms do not incorporate edge feature preservation in their designs and cannot effectively separate edge pixels from other image pixels in their smoothing processes. Thus, either the smoothing result blurs out edge features or the image noise is not effectively removed. The contextual based smoothing algorithms in general have better performance over non-contextual ones with respect to edge preservation and noise removal and the scale based algorithms are superior in performance over the non-scale based algorithms [1,3]. It has been shown in [2] that Chen's algorithm can effectively remove noise while preserving image features. There are three parameters, h, S, and T that are needed to be selected in the algorithm. Parameters $\mathrm{h}$ and $\mathrm{S}$ control the impact of inhomogeneity value $\mathrm{H}$ and the gradient on the smoothing speed respectively and parameter $\mathrm{T}$ is the total number of iterations. While $\mathrm{S}$ is not sensitive to the final results and can set between 10 and $20, \mathrm{~h}$ and $\mathrm{T}$ have to be carefully selected in order to give quality smoothing results. In many of our simulations, SNR drops off rather rapid after T number of iterations. In this paper, an improved algorithm is proposed to further improve edge feature preservation feature and also provide a practical way to select values of parameter $h$ and $T$.

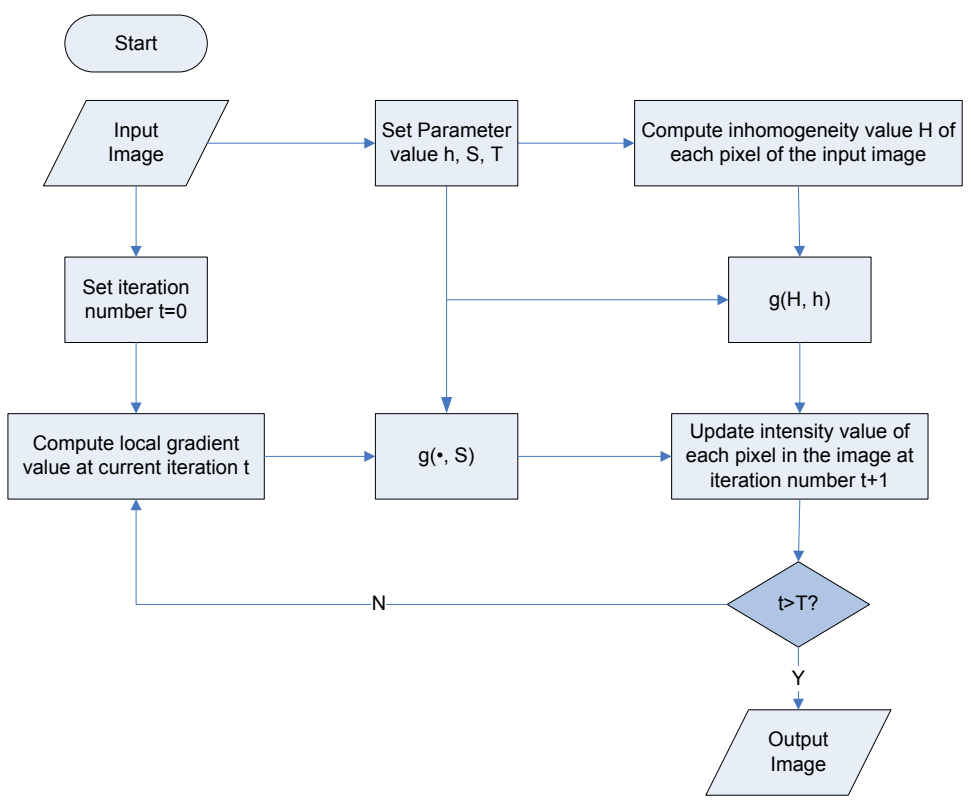

Fig. 1. A functional flowchart of chen's smoothing algorithm

\section{Problem Statement}

As discussed in the introduction section, Chen adaptive smoothing algorithm is conceptually attractive. Our aim is to improve upon his algorithm. Here a brief presentation of Chen's algorithm is provided. It is a pixel based adaptive smoothing algorithm 
where pixels with smoother neighborhood have larger smoothing actions while pixels with rougher neighborhood have smaller smoothing actions. The iterative smoothing action for a pixel $(\mathrm{x}, \mathrm{y})$ is defined as.

$$
I_{(x, y)}^{t+1}=I_{(x, y)}^{t}+\Delta I\left(H_{(x, y)}, \nabla I_{(x, y)}^{t}, \Delta f_{(x, y)}^{t}\right)_{(x, y)}^{t}
$$

The $\Delta I(\cdot)_{(x, y)}^{t}$ determines the amount of smoothing action in a given iteration, and it is jointly determined by inhomogeneity $H_{(x, y)}$, gradient $\nabla I_{(x, y)}^{t}$, and which is the intensity difference of pixel $(\mathrm{x}, \mathrm{y})$ and its 8 adjacent neighbors. The $H_{(x, y)}$ is a measurement of pixel $(\mathrm{x}, \mathrm{y})$ 's contextual discontinuity, and $\nabla I_{(x, y)}^{t}$ is a measurement of its local discontinuity. Both discontinuity measurements are combined to determine the appropriate smoothing action or speed for the pixel. $\mathrm{H}$ is calculated by averaging the disconnectedness values among a pixel and its 8 adjacent pixels where the disconnectedness value between the pixel and one of its neighbors is calculated using a square shape homogeneous neighborhood $(\mathrm{HN})$. Since $\mathrm{HN}$ is restricted to a square shape and often does not represent the actual homogenous area around the pixel. For a smaller neighborhood, the size and shape errors may lead to error in $\mathrm{H}$. The smallest $\mathrm{HN}$ of 3 by 3 may not reflect the true size and shape of the homogeneous area when the adjacent pixel is an edge pixel. Furthermore, $\mathrm{H}$ does not reflect the direction of the edge which may be important when the pixel under consideration is adjacent to an edge feature pixel rather than a noisy pixel. It is rather important that we have a correct $\mathrm{H}$ map to reflect the real contextual discontinuity value of the pixel. In addition, our numerous experiments on real images ( 5 in number) have indicated that the SNR of the resulting image is rather sensitive to the value of parameter $h$ and $T$. The fact that the SNR drops off rather rapidly as the number of iteration increases is rather important. These difficulties are limiting the practical applicability of the algorithm to any noise contaminated image where the original image is not available.

It is obvious that a correct $\mathrm{H}$ map is the key in getting a good smoothing result since the algorithm aggressively smoothing out homogenous regions of an image while at the same time limiting the smoothing operation in edge feature rich regions. In this paper, a modified procedure to compute the $\mathrm{H}$ map is provided. Parameter settings are to be estimated through an experimental searching operation.

\section{Approach}

A good $\mathrm{H}$ map depends on a correct $\mathrm{HN}$ and correct $\mathrm{HN}$ depends on having a correct shape of homogeneity neighborhood which is not a square in general. Here, we propose the following two improvements:

\subsection{Generation of a Correct Homogeneity Neighborhood(HN)}

A correct neighborhood has the following properties: it takes the shape of homogeneous neighborhood and is not necessarily a square one, meets a selected smoothness criterion, and finally, has the largest neighborhood area (larger than 3 by 3 ). The larger is the HN size the better (more accurate) is the statistics on the intensity calculations. The level set method is used to search for the HN since the method is an iterative 
process derived from a selected smoothness criterion and guarantees a connected region from an initial starting local region around a pixel. The smoothness criterion is embedded in the deforming speed function in deforming the HN. The speed function used in our implementation is the commonly adopted inverse of a gradient function. The initial contour is placed around the interested pixel and consists of its 4 neighbors. To reduce the computation time, the maximum number of iterations is set to be 5 , and the maximum size of $\mathrm{HN}$ is set to be 150 pixels. A morphological erosion operation is applied to the resulting close region to produce a smoother $\mathrm{HN}$. $\mathrm{H}$ value of each pixel is the average brightness of the pixels in its HN. Obviously, with this modification, the problem of having 3 by $3 \mathrm{HN}$ in Chen's algorithm is overcome.

\subsection{Incorporation of the Directional Information in the $H$ Map Computation}

The H map is derived from the HN map to extract the inhomogeneity (context discontinuity) of a pixel and its 8 neighboring pixels. As stated earlier, it reflects whether a pixel is adjacent to an edge element or not. The directional information is an important knowledge needed in the adaptive method to identify the neighboring edge pixels as illustrated in Fig. 2. A large average intensity difference between two adjacent pixels' HNs in a particular direction is an indication of either the presence of a large noise pixel or an adjacent edge segment. To distinguish these two situations, a threshold value is used to compare with these directional differences. If three or more adjacent pixels differences exceed this threshold, then the present of an edge segment is declared and smoothing weights are changed accordingly. To accomplish this, $\Delta \mathrm{HN}(\mathrm{i})$ is computed as the average intensity difference of the center pixel's $\mathrm{HN}$ and the $\mathrm{HN}$ of its ith adjacent pixel with i being from 1 to 8 .

$$
\mathrm{H}(*)=\sum \omega_{\mathrm{i}} \Delta \mathrm{HN}(\mathrm{i}) ; \sum \omega_{\mathrm{i}}=1 ; \mathrm{i}=1 \sim 8 ; .
$$

The $\Delta \mathrm{HN}(\mathrm{i})$ indicates the directional intensity difference. As illustrated in Fig.2, the $\Delta \mathrm{HN}(\mathrm{i})$ in the diagonal direction will be larger than all other directions. The weight wi will be set larger to emphasize the directional discontinuity.

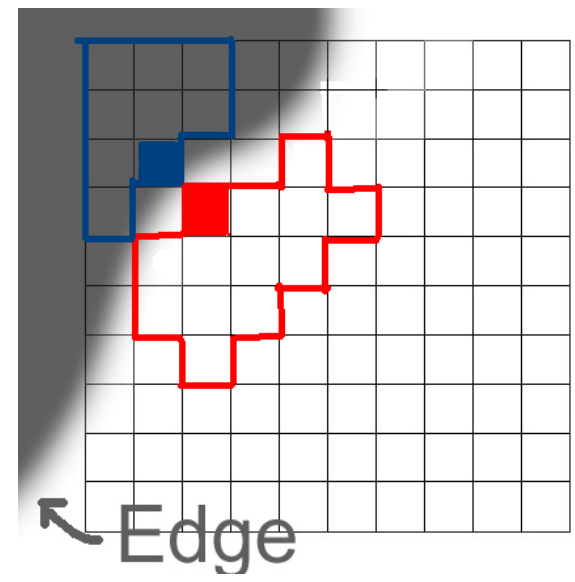

Fig. 2. An illustration of two $\mathrm{HN}$ neighborhoods of the edge 
All the improvements are concentrated in producing an improved $\mathrm{H}$ map so that a better smoothing result can be obtained with edge features being properly preserved. The rest of the adaptive iterative smoothing algorithm stays the same as Chen original algorithm.

\section{Experimental Results}

Experiments are designed to show comparisons of performances of our proposed method and Chen method based on SNR, DR(edge feature detection rate) and FAR(edge feature false alarm rate) on a set of 5 real images (shown in Fig.3) contaminated with various degrees of additive noise. In addition, parameter settings of $h$ and $\mathrm{T}$ are experimentally determined using the 5 real images.

\subsection{Performance Comparison between Our Algorithm and Chen's Algorithm}

Chen's algorithm has been considered to give better performance in terms of SNR than most other adaptive methods. Chen's algorithm is implemented and verified per [3] by us to produce more experiment results beyond reported results. In our experiments, Gaussian noise with zero mean and different standard deviations (Std) is added to an original image. The comparisons are based on two key objectives - high SNR and edge preservation. For edge preservation evaluation, we have devised the following procedure:

i) Use the Sobel edge operator with a threshold to extract all edge pixels from an image.

ii) Use the resulting edge pixels from an original image as the ground truth.

iii) Calculate detection rate (DR) and false-alarm rate (FAR) as measures of the edge preservation capability.

DR and FAR are defined as:

$\mathrm{DR}=($ Total number of edge pixels matched between the smoothed image and the original image) / (Total number of edge pixels of the original image).

FAR $=$ (Total number of edge pixels found in the smoothed image and not in the original image) / (Total number of edge pixels of the original image).

It is desirable that a good smoothing method will have a high DR together with a very low FAR. It is possible for the FAR to be much larger than 1 when the total number of true edge pixels is much smaller than the image size under noisy condition. The main reason of selecting Sobel operator is that it is a simple and non-smoothing edging method. Although different threshold values give different sets of edges, it has been shown in our experiments that the results are consistent when comparing the performances of the two methods using different threshold values (In comparison different threshold values, one same threshold value will be applied in both methods each time).

The 5 images in Fig. 3 are selected to reflect a broader class of images to provide a more critical comparison of performance. These images are carefully selected to reflect different image data collection situations from an object with a smooth to a 

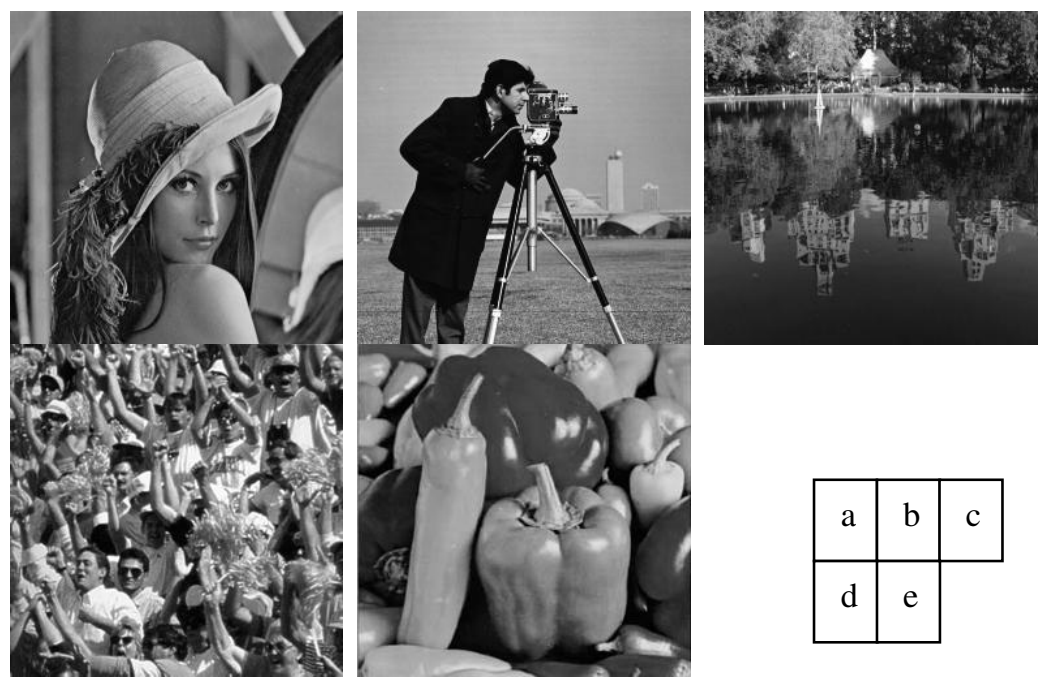

Fig. 3. Images: a) Lena; b) Cameraman; c) Scene; d) Human face; e) Peppers

highly texture image, from a single person image to a very dense crowd, and from an in-door image to an out-door scene. It is expected that the experimental results derived from these images can provide a preliminary and practical conclusion on the applicability of the two algorithms. Additive Gaussian noise of STD of 10 and 20 are considered like most others reported in the literature.

The ground truth edge pixels are extracted using the Sobel operator with a threshold on the original image. Note that different threshold settings give different total number of edge pixels. Our experiments have indicated that the comparison results of performances are consistence using different threshold values(Applying the same threshold value to generate comparing result each time and changing the threshold value to produce a set of comparison results). Be noted that Sobel operator preserves high frequency texture edge features. The results are presented in Table 1 and 2. The results show slight improvements on SNRs and significant improvements on FARs in all 5 cases when comparing the two methods. While our method provides lower DRs when compared to that of original images, they are consistently higher than using the Chen method. Furthermore, the DRs are reduced by $10 \%$ to $20 \%$ due to the smoothing operation with the order of magnitude improvements in the FARs which maybe significant to any further image segmentation operations. Note that some high frequency texture pixels are also eliminated in the process in producing lower DRs, and therefore, the DRs are even lower for more complicated or busy images like Fig.3d and 3e. These Low DR numbers should not affect the true edge pixels for most object segmentation applications since they are non-texture data. We also have the results on an MRI brain image with various degrees of added noise with significant improvements in SNR. We do not have DR and FAR figures in this case. 
Table 1. Smoothing results comparison for 5 real images (without small region removal process)

\begin{tabular}{|l|l|l|l|l|l|l|l|l|l|l|l|}
\hline \multicolumn{2}{|l|}{ Image } & \multicolumn{2}{|c|}{ Fig. 3a } & \multicolumn{2}{l|}{ Fig. 3b } & \multicolumn{2}{l|}{ Fig. 3c } & \multicolumn{2}{|c|}{ Fig. 3d } & \multicolumn{2}{c|}{ Fig. 3e } \\
\hline \multicolumn{2}{|l|}{ Test Setting } & $*$ & $* *$ & $*$ & $* *$ & $*$ & $* *$ & $*$ & $* *$ & $*$ & $* *$ \\
\hline \multirow{4}{*}{ A } & SNR & 125 & 32 & 185 & 48 & 63 & 17 & 170 & 43 & 272 & 71 \\
\cline { 2 - 14 } & DR & 0.93 & 0.91 & 0.85 & 0.83 & 0.83 & 0.84 & 0.89 & 0.84 & 0.95 & 0.92 \\
\cline { 2 - 14 } & FAR & 1.7 & 2.17 & 0.07 & 0.65 & 0.12 & 0.74 & 0.17 & 0.24 & 0.0 & 1.1 \\
\hline \multirow{3}{*}{ B } & SNR & 220 & 139 & 456 & 185 & 121 & 82 & 224 & 117 & 443 & 267 \\
\cline { 2 - 12 } & DR & 0.68 & 0.58 & 0.69 & 0.58 & 0.68 & 0.5 & 0.68 & 0.63 & 0.81 & 0.73 \\
\cline { 2 - 12 } & FAR & 0.11 & 0.14 & 0.05 & 0.13 & 0.1 & 0.08 & 0.08 & 0.1 & 0.12 & 0.32 \\
\hline C & SNR & 248 & 149 & 462 & 199 & 128 & 87 & 243 & 129 & 454 & 281 \\
\cline { 2 - 11 } & DR & 0.79 & 0.63 & 0.75 & 0.62 & 0.7 & 0.52 & 0.73 & 0.69 & 0.86 & 0.74 \\
\cline { 2 - 11 } & FAR & 0.09 & 0.10 & 0.02 & 0.03 & 0.04 & 0.05 & 0.03 & 0.04 & 0.06 & 0.09 \\
\hline
\end{tabular}

*: Gaussian noise with 0 mean and std 10 ; **: Gaussian noise with 0 mean and std 20 .

A: Noisy image; B: Smoothed image with Chen's method; C: Smoothed Image with our method.

Table 2. Smoothing results comparison for 5 real images (with small region removal process)

\begin{tabular}{|l|l|l|l|l|l|l|l|l|l|l|l|}
\hline \multicolumn{2}{|l|}{ Image } & \multicolumn{2}{|c|}{ Fig. 3a } & \multicolumn{2}{c|}{ Fig. 3b } & \multicolumn{2}{c|}{ Fig. 3c } & \multicolumn{2}{c|}{ Fig. 3d } & \multicolumn{2}{c|}{ Fig. 3e } \\
\hline \multicolumn{2}{|l|}{ Test Setting } & $*$ & $* *$ & $*$ & $* *$ & $*$ & $* *$ & $*$ & $* *$ & $*$ & $* *$ \\
\hline \multirow{4}{*}{ A } & SNR & 125 & 32 & 185 & 48 & 63 & 17 & 170 & 43 & 272 & 71 \\
\cline { 2 - 14 } & DR & 0.94 & 0.93 & 0.93 & 0.88 & 0.89 & 0.86 & 0.93 & 0.88 & 0.96 & 0.93 \\
\cline { 2 - 13 } & FAR & 1.76 & 1.9 & 0.17 & 0.82 & 0.25 & 1.04 & 0.17 & 0.37 & 0.81 & 0.98 \\
\hline \multirow{3}{*}{ B } & SNR & 220 & 139 & 456 & 185 & 121 & 82 & 224 & 117 & 443 & 267 \\
\cline { 2 - 12 } & DR & 0.73 & 0.6 & 0.8 & 0.64 & 0.77 & 0.56 & 0.75 & 0.70 & 0.84 & 0.75 \\
\cline { 2 - 12 } & FAR & 0.09 & 0.11 & 0.11 & 0.2 & 0.09 & 0.12 & 0.1 & 0.2 & 0.1 & 0.21 \\
\hline C & SNR & 248 & 149 & 462 & 199 & 128 & 87 & 243 & 129 & 454 & 281 \\
\cline { 2 - 11 } & DR & 0.81 & 0.67 & 0.86 & 0.72 & 0.79 & 0.58 & 0.79 & 0.74 & 0.88 & 0.77 \\
\cline { 2 - 11 } & FAR & 0.07 & 0.08 & 0.06 & 0.06 & 0.08 & 0.1 & 0.04 & 0.09 & 0.05 & 0.08 \\
\hline
\end{tabular}

*: Gaussian noise with 0 mean and std 10; **: Gaussian noise with 0 mean and std 20.

A: Noisy image; B: Smoothed image with Chen's method; C: Smoothed Image with our method.

\subsection{Experimental Determination of $h$ and $T$ Parameter Values}

The main purpose of the experiment is to enhance the applicability of our proposed smoothing method and Chen method. Both methods require the selections of two significant parameter values - "h" and "T" (the total iteration number). Experiments have shown the resulting smoothed image is not sensitive to selection of $S$ value as long as it stays within the range between 10 and 40. If we can show experimentally that the smoothing results do not change very much in SNRs, i.e., SNR figures are within a narrow band, for ranges of $h$ and $T$ will improve the applicability of our proposed method. Be noted that on real images, the SNRs drop off rather rapidly after $\mathrm{T}$ iterations. Our experimental approach is to do an exhaustive search of $h$ and $T$ in obtaining the best SNR figures of all 5 images under the contamination of an additive Gaussian noise. 


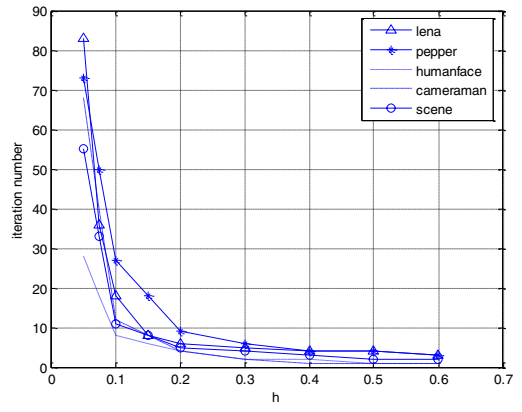

(a)

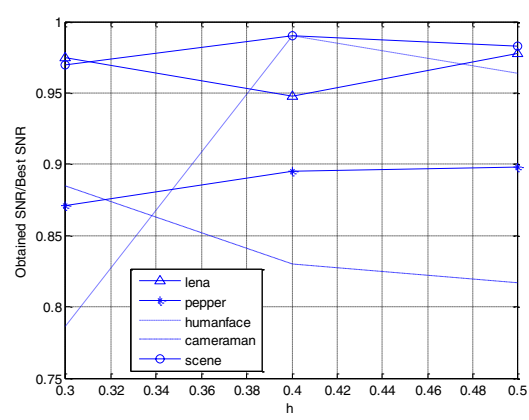

(b)

Fig. 4. Our algorithm, (a): besting stopping iteration number versus h; (b): obtained SNR/best SNR versus $h$

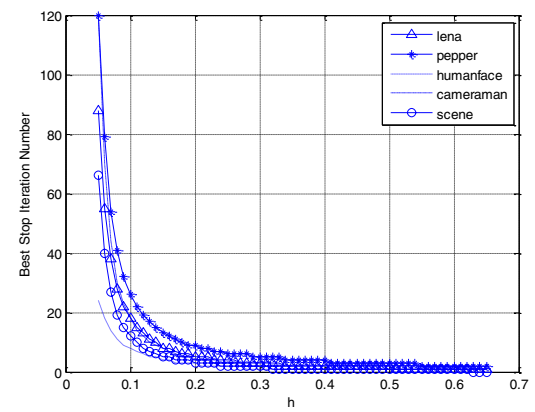

(a)

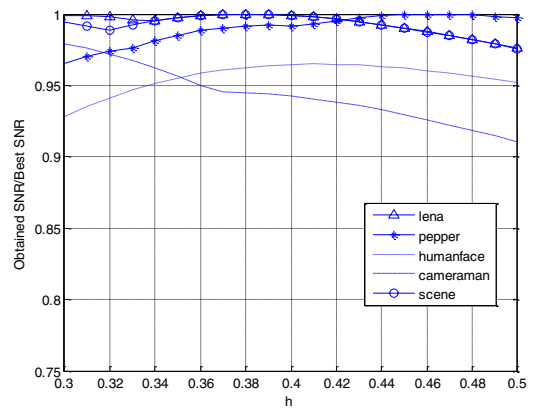

(b)

Fig. 5. Chen's algorithm, (a): besting stopping iternation number versus h; (b): obtained SNR/best SNR versus $h$

For all 5 images in Fig.3, SNRs are computed for different values of h and $\mathrm{T}$ with each incoming image being contaminated with Gaussian noise of standard deviation of 20. Our experiments show that the SNR peaks for higher $h$ values with smaller $\mathrm{T}$ values, and they drop off rather rapidly for larger $h$ and larger $T$. For parameter value range selection results, we have Fig.4a and Fig.4b. Fig.4a shows the plots of the T (number of iterations for the best SNR) versus the h. One can see that T is less than 8 iterations in all 5 images for $\mathrm{h}$ ranging from 0.2 to 0.6 . Fig. $4 \mathrm{~b}$ shows the plot ratios of the obtained SNR versus best SNR figure (maximum of 1) for each image versus $h$ ranging from 0.3 to 0.5 . There is only one best SNR for each image. While the resulting SNRs are better than $80 \%$ of the best SNR, and they stay rather close range the SNR performances for the whole range of $h$. Therefore, one can draw a conclusion that for these 5 images, and for $\mathrm{T}$ ranges from 3 to 6 and $\mathrm{h}$ ranges from 0.3 to 0.5 , our smoothing method will provide SNRs of over $80 \%$ of the best SNRs. After the computation of the $\mathrm{H}$ map, one needs only a very few iterations to obtain a smoothed image in practice as shown in these 5 images. We also conduct the same experiments using Chen's algorithm and very similar results for $\mathrm{h}$ and $\mathrm{T}$ are shown in Fig.5a and 5b. 


\section{Conclusion}

Our modified algorithm shows slight improvements in SNR when compared to that of Chen's algorithm as can be seen in Tables 1 and 2 for 5 selected images shown in Fig. 3 with different degrees of added noise. These tables show also large improvements in preserving edge features when comparing the same two algorithms. Furthermore, experimental results shown in Fig. 4 and 5 allow us to select the two key parameters of $\mathrm{h}$ ranging from 0.3 to 0.5 and T ranging from 3 to 6 that provide comparable smoothing results (higher than $80 \%$ from the best SNR) in all 5 images shown in Fig. 3 for both our algorithm and Chen's algorithm. The best combination set for any specific application can visually be determined experimentally using a small number of combinations of different $h$ and T. For practical applications without the knowledge of the noise free image and/or a viewer, we suggest the set of $\mathrm{h}=0.4$ and $\mathrm{T}=4$.

\section{Acknowledgement}

The authors wish to thank Dr. Ke Chen for clarifying his smoothing algorithm and also for providing the source code.

\section{References}

1. Udupa, J.K., Samarasekera, S.: Fuzzy Connectedness and Object Definition: Theory, Algorithms, and Applications in Image Segmentation. Graphical Models Image Processing 58(3), 246-261 (1998)

2. Chen, K.: Adaptive Smoothing via Contextual and Local Discontinuities. IEEE Trans. on Pattern Analysis and Machine Intelligence 27(10), 1552-1567 (2005)

3. Gonzalez, R.C., Woods, R.E.: Digital Image Processing, 2nd edn. Prentice Hall, Upper Saddle River (2002)

4. Perona, P., Malik, J.: Scale-Space and Edge Detection Using Anisotropic Diffusion. IEEE Trans. Pattern Analysis and Machine Intelligence 12, 629-639 (1990)

5. Black, M.J.: Robust Anisotropic Diffusion. IEEE Trans. Image Processing 7, 421-432 (1998)

6. Saha, P.K., Udupa, J.K.: Scale-Based Diffusive Image Filtering Preserving Boundary Sharpness and Fine Structures. IEEE Trans. Medical Imaging 20, 1140-1155 (2001)

7. Chen, K.: A feature preserving adaptive smoothing method for early vision. Journal of Pattern Recognition Society 13 (2000)

8. Saha, P.K., Udupa, J.K.: Scale-Based Fuzzy Connected Image Segmentation: Theory, Algorithms, and Validation. Computer Vision and Image Understanding 77, 145-174 (2000)

9. Saha, P.K., Udupa, J.K.: Scale-based filtering of medical images. In: Proc. SPIE: Medical Imaging, vol. 3979, pp. 735-746 (2000)

10. Saha, P.K., Udupa, J.K.: Optimum Image Thresholding via Class Uncertainty and Region Homogeneity. IEEE Trans. Pattern Analysis and Machine Intelligence 23, 689-706 (2001)

11. Pednekar, A.S., Kakadiaris, I.A.: Image Segmentation Based on Fuzzy Connectedness Using Dynamic Weights. IEEE Transaction on Image Processing 15(6), 1555-1562 (2006) 
12. Vu, R.H.: Fuzzy algorithms: Application to adipose tissue quantification on MR images. Biomedical Signal Processing and Control 2(3), 239-247 (2007)

13. Peng, H.: Modification and Implementation of a 2D Context-Sensitive Adaptive Smoothing Algorithm and its Extension to 3D, master thesis in University of Cincinnati (2006)

14. Hou, Y.: Application of a 3D Level Set Method in MRI Surface Segmentation, Master thesis in University of Cincinnati (2005)

15. Sethian, J.A.: Level Set Methods and Fast Marching Methods: Evolving Interfaces in Computational Geometry, Fluid Mechanics, Computer Vision and Materials Science. Cambridge University Press, Cambridge (1999)

16. McInerney, T., Terzopoulos, D.: Deformable models in medical image analysis: a survey. Medical Image Analasis 1, 91-108 (1996)

17. Pednekar, A.S., Kakadiaris, I.A.: Image Segmentation Based on Fuzzy Connectedness Using Dynamic Weights. IEEE Transaction on Image Processing 15(6), 1555-1562 (2006)

18. Herman, G.T., Carvalho, B.M.: Multiseeded segmentation using fuzzy connectedness. IEEE Trans. on Pattern Anal. Mach. Intell. 23, 460-474 (2001)

19. Vu, R.H.: Fuzzy algorithms: Application to adipose tissue quantification on MR images. Biomedical Signal Processing and Control 2(3), 239-247 (2007)

20. Bloch, I.: Fuzzy spatial relationships for image processing and interpretation: a review. Image and Vision Computing 23(2), 89-110 (2005) 